\title{
СОВРЕМЕННЫЕ ПОДХОДЫ К ЛЕЧЕНИЮ ОСТРЫХ ЯЗВ ПИЩЕВАРИТЕЛЬНОГО КАНАЛА, ОСЛОЖНЕННЫХ КРОВОТЕЧЕНИЕМ, У БОЛЬНЫХ ПОСЛЕ ОПЕРАЦИЙ НА ОРГАНАХ ГЕПАТОПАНКРЕАТОБИЛИАРНОЙ ЗОНЫ
}

\author{
В. В. Бойко, В. Н. Лыхман, С. В. Ткач, А. А. Меркулов, Д. Ю. Гуляева, Е. В. Ханько \\ Институт общей и неотложной хирургии имени В. Т. Зайцева НАМН Украины, г. Харьков
}

\section{MODERN APPROACH TO THE MANAGEMENT OF ACUTE ULCERS OF DIGESTIVE TRACT COMPLICATED BY BLEEDING IN PATIENTS AFTER HEPATOBILIAR ZONE SURGERY}

\author{
V. V. Boyko, V. N. Lykhman, S. V. Tkach, A. A. Merkulov, D. Yu. Guliaieva, Ye. V. Khanko \\ Zaitsev Institute of General and Emergency Surgery, Kharkov
}

\section{Рефрерат}

Представлены результаты лечения больных по поводу острых язв желудка и двенадцатиперстной кишки (ДПК), осложненных кровотечением, возникших после операций на органах гепатопанкреатобилиарной зоны. Внедрение разработанной индивидуализированной тактики лечения пациентов с активным использованием современных методов эндогемостаза позволило улучшить его результаты.

Ключевые слова: острые язвы желудка и двенадцатиперстной кишки; кровотечение; хирургическое лечение.

Abstract

The results of management of patients with acute gastric and duodenal ulcers complicated by bleeding after hepatobiliar zone surgery are given. Implementation of individualized management with application of up-to-date endohemostatic procedures significantly improved the results.

Keywords: acute gastric and duodenal ulcers; bleeding; surgical treatment.

Несмотря на использование современных консервативных методов профилактики язвенного поражения в послеоперационном периоде, в последние десятилетия наблюдают увеличение числа больных, у которых после различных оперативных вмешательств возникают острые язвы желудка и ДПК, осложненные кровотечением [1 - 3]. Острое эрозивно-язвенное поражение желудка и ДПК выявляют у 2,8\% больных после операций на сердце и крупных сосудах, у 67\% - после обширных операций на органах брюшной полости. Тяжелым осложнением острых язв является кровотечение, возникающее у 30\% больных в основном в раннем послеоперационном периоде, когда на больного воздействуют агрессивные факторы, обусловленные операцией [4].

Летальность при кровотечении из острых язв достигает 35 - 80\%, в зависимости от возраста пациентов, тяжести сопутствующих заболеваний [5 - 7].

Основным патогенетическим механизмом эрозивно-язвенного по- ражения является преобладание факторов внутрижелудочной агрессии по отношению к факторам защиты $[1,2]$. В ближайшем послеоперационном периоде повышение кислотопродукции происходит на фоне угнетения моторики желудка. При нарушении эвакуации кислого содержимого желудка кислотнопептический фактор длительно воздействует на слизистую оболочку желудка, что в сочетании с другими причинами значительно повышает риск язвообразования. Реакция пищеварительной системы на хирургический стресс лежит в основе образования ранних истинных стрессовых язв, частота которых составляет примерно 80\% всех изъязвлений слизистой оболочки верхних отделов пищеварительного канала, образующихся в послеоперационном периоде. При заболеваниях органов гепатопанкреатобилиарной зоны, особенно осложненных обтурационной желтухой, часто после операции значительно активизируется секреция желудка, что способствует образованию острых язв [1].
Критерии выбора оптимальной тактики лечения острых язв желудка и ДПК, осложненных кровотечением, у больных после обширных операций на органах гепатопанкреатобилиарной зоны окончательно не определены.

Цель исследования: улучшение результатов лечения острых язв желудка и ДПК, осложненных кровотечением, возникших после оперативных вмешательств по поводу заболеваний органов гепатопанкреатобилиарной зоны.

\section{МАТЕРИАЛЫ И МЕТОДЫ ИССЛЕДОВАНИЯ}

Проанализированы результаты лечения в клинике 47 пациентов в период с 2009 по 2016 г., которым произведены различные оперативные вмешательства на органах гепатопанкреатобилиарной зоны. После операции у них образовались острые язвы желудка и ДПК, которые проявлялись желудочно-кишечным кровотечением (ЖКК) различной тяжести. Мужчин было 29 (61,7\%), женщин - 18 (38,3\%). Возраст паци- 
ентов от 36 до 82 лет, 68\% больных были пожилого и старческого возраста. Из сопутствующих заболеваний у 63\% пациентов диагностированы сердечно-сосудистые заболевания на фоне мультифокального атеросклероза, у 23\% - цереброваскулярная болезнь, у 21\% - сахарный диабет, у 12 (25,5\%) - желчнокаменная болезнь, осложненная холедохолитиазом и обтурационной желтухой, у 5 (10,6\%) - гепатоцеллюлярная карцинома, у 2 (4,2\%) - холангиоцеллюлярная карцинома, у 10 (21,3\%) - метастатический колоректальный рак, у 4 (8,5\%) - рак поджелудочной железы, у 4 (8,5\%) - фиброзно-кистозные дегенеративные осложнения хронического панкреатита, у 10 (21,3 \%) - панкреонекроз.

Холецистэктомия, холедохолитотомия, наружное дренирование общего желчного протока (ОЖП) по Вишневскому выполнены у 12 больных, холецистоеюностомия - у 2, правосторонняя расширенная гемигепатэктомия - у 6, резекция ОЖП, левосторонняя гемигепатэктомия у 1 , левосторонняя секциоэктомия у 5, левосторонняя гемиколэктомия, сегментэктомия - у 5, дистальная панкреатэктомия - у 2, лапаротомия, некрсеквестрэктомия - у 5, видеоассистированная люмботомия, некрсеквестрэктомия - у 5, лапаротомия, продольная панкреатикоеюностомия - у 4.

Предложенный лечебно-диагностический алгоритм включал: на первом этапе с учетом оценки тяжести состояния больного, вероятной причины возникновения и тяжести ЖКК выполнение в неотложном порядке эзофагогастродуоденофиброскопии (ЭГДФС). Во время исследования оценивали локализацию язвенного дефекта, его размеры, глубину, наличие в момент осмотра активного кровотечения; при активном кровотечении вторым этапом применяли эндоскопические методы гемостаза, оценивали риск рецидива кровотечения по классификации D. Forrest (1987). Наряду с инъекционным гемостазом, использовали коагуляционный гемостаз и эндоклиппирование. При эффективности гемостаза и низком риске рецидива ЖКК проводили консер- вативную терапию с последующей контрольной ЭГДФС.

При высоком риске рецидива ЖКК (FIa, FIb, FIIa, FIIb) и наличии признаков прогрессирования полиорганной недостаточности (ПОН) проводили контрольную ЭГДФС на 3, 5-е, 8-е сутки после достижения первичного гемостаза. Для объективной оценки тяжести состояния больных в первые сутки возникновения кровотечения использовали балльную систему АРАCHЕ III (Acute Physiological and Chronic Health Estimation). Профилактика рецидивов кровотечения из острых язв включала не только проведение соответствующей антисекреторной терапии, но и лечение ПОН, стабилизацию состояния больного.

\section{РЕЗУЛЬТАТЫ \\ И ИХ ОБСУЖДЕНИЕ}

У 44,7\% пациентов при первичной эндоскопии наблюдали гематин в дне язвы, что соответствовало FIIс и не сопровождалось высоким риском рецидива кровотечения. При активном ЖКК из язвы желудка или ДПК (FIa, FIb) применяли все доступные методы эндогемостаза. При состоявшемся ЖКК, когда язвенный кратер закрыт свежим сгустком крови (FIIa) и гемостаз был ненадежным, препараты вводили в подслизистый слой вокруг язвы, стараясь не повредить кровяной сгусток. В 70\% наблюдений применен инъекционный гемостаз (изотонический раствор натрия хлорида с этоксисклеролом 0,5 мл 2\% в качестве склерозанта); в 20\% - коагуляционный гемостаз (монополярный, биполярный, с применением термозонда и электрохирургических щипцов); в 10\% - во время первичной эндоскопии проведено клиппирование кровоточащего сосуда. Наряду с использованием методик эндоскопического гемостаза проводили консервативную терапию, назначали ингибиторы протонной помпы (ИПП), использование которых позволяет уменьшить риск рецидива ЖКК и необходимость выполнения хирургического вмешательства [7]. Эффективность первичного эндоскопического гемостаза с использованием современных методов со- ставила 89\%, у 11\% больных при неэффективности эндоскопического гемостаза и высоком риске (3 4 степени) рецидива ЖКК выполнено оперативное вмешательство. При неэффективности или невозможности осуществления эндоскопического гемостаза некоторые авторы рекомендуют выполнять ангиографию [8, 9]. При стрессовых эрозиях и язвах источник кровотечения в 95\% наблюдений расположен в проксимальном отделе желудка, который на 85\% кровоснабжается из левой желудочной артерии. Поэтому применяют селективную ангиографию с введением сосудосуживающих препаратов в левую желудочную артерию или ее эмболизацию. у 3 (6,4\%) больных использованы рентгеноэндоваскулярные методики гемостаза в виде эмболизации левой желудочной артерии, достигнут надежный гемостаз.

У 2 (4,3\%) больных при панкреонекрозе и ПОН возникло профузное кровотечение, выполнена «реанимационная лапаротомия». При профузном язвенном кровотечении в клинике применяем интраоперационный многокомпонентный ступенчатый гемостаз, временный и окончательный [4]. Анестезиологическое пособие и периоперационная интенсивная терапия включали интубацию трахеи на самостоятельном дыхании после введения кетамина в дозе 2 - 4 мг/кг; введение эритроцитсодержащих препаратов крови (только после хирургического гемостаза). Иссечение язвы ДПК (субциркулярное) выполнено у 1 больного, с ее частичной экстериоризацией - у 1 .

При тяжелых заболеваниях органов гепатобилиарной системы, образовании острых язв желудка и ДПК, осложненных кровотечением, после выполнения обширного оперативного вмешательства общая летальность составила 6,4\%. Вследствие печеночной недостаточности и ПОН умерли 2 (4,2\%) больных, острой сердечно-сосудистой недостаточности - 1 (2,1\%).

Таким образом, внедрение разработанного лечебно-диагностического алгоритма с активным использованием современных методов эн- 
догемостаза и ИПП у пациентов по поводу острых язв, возникших после операций на органах гепатопанкреатобилиарной зоны, позволило из- бежать рецидивов ЖКК, уменьшить общую летальность до 6,4\%.

\section{ЛИТЕРАTYPA/REFERENCES}

1. Kubyshkin VA, Shishin KV. Erozivno-yazvennoe porazhenie verkhnikh otdelov zheludochno-kishechnogo trakta v rannem posleoperatsionnom periode. Khirurgiya. 2004;(4):8-9.

2. Nikishaev VI, Zadorozhnyy AM. Profilaktika povtornykh retsidivov krovotecheniya u bolnykh s sindromom Mellori-Veysa. Ukrainskyi zhurnal maloinvazyvnoi ta endoskopichnoi khirurhii. 2007;4(11):32.

3. Lee JW, Kim HK, Woo YS, Jahng J, Jin YR, Park JH, et al. Optimal operational definition of patient with peptic ulcer bleeding for big data analysis using combination of clinical characteristics in a secondary general hospital. Korean J Gastroenterol. 2016;68(2):77-86.

4. Constantin VD, Paun S, Ciofoaia VV, Budu V, Socea B. Multimodal management of upper gastrointestinal bleeding caused by stress gastropathy. J Gastrointest Liver Dis. 2009 Sep;18(3):279-84.

5. Boyko VV, Sushkov SV, Krivorot'ko IV, Dotsenko EG, Peev SB, Tkachuk AYU, Kozlova TV. «Reanimatsionnye» laparotomii pri profuznom krovotechenii iz verkhnikh otdelov zheludochno-kishechnogo trakta. Ukrainskyi zhurnal khirurhii. 2011;4(13).

6. Velihotskyi MM, Velihotskyi OM, Komarchuk VV, Horbulich OV, Smetskoi DO, Kobyletskyi MI, Lazutkina OA. Indyvidualna-aktyvna taktyka, prohnoz i modyfikatsiia orhanozberihaiuchykh operatsii pry vyrazkovykh krovotechakh. AML XXI. 2015;(4):32-7.

7. Barkun AN, Bardou M, Kuipers EJ, et al. International Consensus Recommendations on the Management of Patients With Nonvariceal Upper Gastrointestinal Bleeding. Ann Intern Med. 2010;152(2):10113.

8. Katano T, Mizoshita T, Senoo K, Sobue S, Takada H, Sakamoto T, et al. The efficacy of transcatheter arterial embolization as the firstchoice treatment after failure of endoscopic hemostasis and endoscopic treatment resistance factors. Dig Endosc. 2012;24(5):364-9.

9. Wong TC, Wong KT, Chiu PW, Teoh AY, Yu SC, Au KW, Lau JY. A comparison of angiographic embolization with surgery after failed endoscopic hemostasis to bleeding peptic ulcers. Gastrointest Endosc. 2011:73(5):900-8. 